\title{
Welcoming the First Decade for Perovskite Solar Cells
}

\author{
Yuanyuan Zhou, ${ }^{1, *}$ Michael Saliba, ${ }^{2, *}$ Iván Mora-Seró $3,{ }^{3}$
}

\footnotetext{
${ }^{1}$ School of Engineering, Brown University, Providence, Rhode Island 02912, USA

${ }^{2}$ Institute of Advanced Materials (INAM), Universitat Jaume I, Av. Sos Baynat, s/n, 12071 Castello, Spain

${ }^{3}$ Technical University Darmstadt, Germany

E-mail: yuanyuan_zhou@brown.edu (Y.Z.); michael.saliba@opto.tu-darmstadt.de (M.S.); sero@uji.es (I.M.-S.)
}

The swift emergence of perovskite solar cells (PSCs) is a 'miracle development' in the history of photovoltaics that has revolutionized the optoelectronic field. Since Miyasaka and coworkers (Toin University of Yokohama, Japan) reported the first use of halide perovskites (HPs) in solar cells in 2009, ${ }^{1}$ the past ten years have witnessed a skyrocketing increase in power conversion efficiency (PCE) of PSCs from $3.8 \%$ to $24.2 \%$ and even $28 \%$ for perovskite on silicon tandems. ${ }^{2}$ Meanwhile, the stability, the Achiles heel of this techniology, has been enhanced very substantially. While the early versions of PSCs were stable just for a few days, the state-of-the-art PSCs have now demonstrated more than 10,000 hours operational stability and a few thousand hours outdoor stability, advancing the practical deployment of PSCs significantly. ${ }^{3,4}$ Such impressive progress in the PCE and stability of PSCs has been not only attributed to the creative technological innovation in device structures, perovskite processing, charge-transport-layer engineering, interface modification, etc., but also related to the fascinating understanding of fundamental sciences in HP materials that include crystal structure, defect chemistry, crystallization, photophysics, and light-matter interaction.

In this context, an important, fruitful discussion on both fundamental and technological progresses in HPs, PSCs, and the broader perovskite optoelectronics was performed in the perovskite-focused 'twin'symposia of ET04: Perovskite Solar Cells - Challenges and Opportunities and ET05: Fundamental Aspects of Halide Perovskite (Opto)electronics and Beyond at the 2018 Materials Research Society (MRS) Fall Meeting (Nov. 26-30, 2018, Boston, MA, USA) which were summarized earlier. ${ }^{5}$ Here, we introduce a collection of papers from the leading scientists from the twin symposia where reporting their most recent research progress and critical opinions on PSCs. We have also invited worldwide contributions from other outstanding perovskite research groups (China, United States, Germany, Sweden, Switzerland, Israel, Brazil, etc.). All these contributions constitute this special issue of Perovskite Photovoltaics and Optoelectronics of Solar $R R L$, that we are delighted to put together for the $10^{\text {th }}$ anniversary celebration of perovskite photovoltaics.

This special issue has had a great reception by the researchers and numerous contributions have been received and consequently will be divided into two part. Here, we introduce the first part that collects 5 review- and 25 research-type articles. For the 5 review-type contributions, (i) Wojciechowski et al. discussed the industrial opportunities of PSCs; (ii) Yin et al. and Pang et al. reviewed the progresses of PSCs based on formamidinium-based HPs and inorganic $\mathrm{CsPbIr}_{2} \mathrm{HPs}$, respectively; (iii) Liu et al. summarized the strategies for making high-performance tin-based PSCs; (iv) Fan et al. provided prospects on environmentally-friendly and stable lead-free perovskites. Regarding the research-type contributions, the following key topics are covered. 
Tailoring perovskite properties and stability. Yan et al. reported the use of block copolymers for grain boundary passivation in $\mathrm{MA}_{0.7} \mathrm{FA}_{0.3} \mathrm{PbI}_{3} \mathrm{HPs}$. Ma et al. used bifunctional dye molecules for enhancing inorganic $\mathrm{Cs} \mathrm{PbIBr}_{2} \mathrm{HPs}$ properties and stability. Zhao et al. showed black phosphorus can promote the photo-stability of $\mathrm{MAPbI}_{3} \mathrm{HPs}$. Chen et al. and Liu et al. demonstrated new perovskite composition engineering strategies. Etgar et al. showed how to tailor the optical properties in already crystallized HPs. Flávia Nogueira et al. revealed the mechanisms underlying the property enhancement of 3D HPs via layered HPs incorporation.

Improving charge-transporting layers and electrodes. Various new types of electron-transporting layers for high-performance PSCs were demonstrated (water-based $\mathrm{TiO}_{2}$ nanocrystals Zhao et al., UVtreated $\mathrm{SnO}_{2}$ by Mora-Seró et al., $\gamma-\mathrm{Ga}_{2} \mathrm{O}_{3}$ nanocrystals by Wang et al., Co-doped $\mathrm{TiO}_{2}$ by Liu et al., $\mathrm{PCBM}$ /conjugated polymer composite by Yang et al., and molecular-engineered PCBM by Troshin et al.). New hole-transporting materials (HTMs) were also designed by Z. Xu et al., Yip et al., B. Xu et al., Getautis et al., and Nazeeruddin et al., which may mitigate the existing issues associated with the commonly-used Spiro-OMeTAD HTMs. Furthermore, Huang et al. and Lin et al. showed the promise of sputtered Au and carbon quantum-dots as conducting contacts for PSCs, respectively.

Modifying device interfaces. Unger et al. and Song et al. demonstrated alkali salts and Zwitterionic polymers for interface modification in PSCs, respectively. An interesting in-situ interface-modification approach was demonstrated by $\mathrm{Hu}$ et al. for enhancing the PSC stability.

Developing lead-free perovskites. Yao et al. showed a new vapor-assisted solution method for making sulfur-incorporated bismuth-based lead-free PSCs. Jen et al. employed trihydrazine-dihydriodide additive for boosting formamidinium tin iodide PSCs to $8.48 \%$ PCE.

In closing, this collection of papers have demonstrated the most recent progress in understanding and developing PSCs from various scientific and technological aspects. We envision they will bring broad attention from the field of photovoltaics and stimulate more significant effort towards more efficient, stable, eco-friendly PSCs in the future. We are also looking forward to releasing the second part of the special issue of Perovskite Photovoltaics and Optoelectronics later this year, and to share more research progress and opinions on perovskite sciences and technologies.

\section{Acknowledgements}

Y.Z. acknowledge support from the National Science Foundation (OIA-1538893) and the Office of Naval Research (N0014-17-1-2232). I.M.-S. thank the European Research Council (ERC) via Consolidator Grant (724424 - No-LIMIT).

Received: ((will be filled in by the editorial staff))

Revised: ((will be filled in by the editorial staff)) Published online: ((will be filled in by the editorial staff))

\section{References}

1. A. Kojima, K. Teshima, Y. Shirai, T. Miyasaka, J. Am. Chem. Soc. 2009, 131, 6050.

2. NREL Best Research-Cell Efficiency Chart. https://www.nrel.gov/pv/cell-efficiency.html.

3. G. Grancini, C. Roldán-Carmona, I. Zimmermann, E. Mosconi, X. Lee, D. Martineau, S. Narbey, F. 
Oswald, F. De Angelis, M. Graetzel, Nat. Commun. 2017, 8, 15684.

4. Y. Rong, Y. Hu, A. Mei, H. Tan, M.I. Saidaminov, S.I. Seok, M.D. McGehee, E.H. Sargent, H. Han, Science 2018, 361, eaat8235.

5. J. Gong, M. Flatken, A. Abate, J.-P. Correa-Baena, I. Mora-Seró, M. Saliba, Y. Zhou, ACS Energy Lett. 2019, 4, 861. 